\begin{tabular}{|l|l|c|c|}
\hline Eiszeitalter u. Gegenwart & 40 & $\begin{array}{c}139-147 \\
6 \mathrm{Abb} .\end{array}$ & Hannover 1990 \\
\hline
\end{tabular}

\title{
Die Kombination geomorphologischer und prähistorischer Arbeitsmethoden bei der Lösung paläogeographischer Fragen in der Eastern Desert Ägyptens
}

\author{
Andreas DitTmanN *)
}

\begin{abstract}
Landform evolution, ephemeral streams, pediment, terraces, travertine, charcoal, ${ }^{14} \mathrm{C}$, absolute age, archaeological sites, artifacts, Middle Paleolithic, Neolithic
\end{abstract}

Egypt, Eastern Desert, Wadi Deir

\begin{abstract}
Kurzfassung: Die nördliche Eastern Desert Ägyptens ist gekennzeichnet durch eine Vielzahl flächenhaft verbreiteter, teilweise ineinandergreifender Systeme von Fußflächen und Wadisedimenten. Bei der Suche nach Möglichkeiten einer relativen Datierung von Entstehung und Entwicklung dieser Geländeformen kommt dem Zusammenwirken prähistorischer und geomorphologischer Arbeitsmethoden eine besondere Bedeutung zu.
\end{abstract}

Beispielhaft für die geomorpho-dynamischen Prozesse dieses Raumes während der letzten 30.000 Jahre werden hier Ergebnisse aus dem Bereich des Wadi Deir an der SüdostAbdachung des Gebel-Galala-el-Qibliya vorgestellt. Innerhalb dieses Untersuchungsgebietes konnten prähistorische Besiedlungsspuren aus mittelpaläolithischer, neolithischer und protohistorischer Zeit nachgewiesen werden. Dabei handelt es sich meist um Oberflächenfunde von Werkzeugen oder Werkplätzen sowie um Felsgravierungen. Darüberhinaus weisen Sinterdatierungen auf die Existenz einer klimatischen Gunstperiode um etwa 26.000 B.P. hin und ${ }^{14} \mathrm{C}$-Datierungen verschiedener Holzkohlehorizonte aus einem in jüngeren Wadisedimenten ausgebildeten Abri belegen hier menschliche Besiedlung zwischen 3.600 und 1.900 B. C.

[The Cooperation of Geomorphologic and Prehistoric Studies Concerning Paleogeographic Investigations in the Eastern Desert of Egypt]

Abstract: The northern parts of the Egyptian Eastern Desert are characterized by a multitude of widespread, partly interlocked systems of pediments and wadi-sediments. In search of possibilities for relative dating of the origin and development of these geomorphologic forms, the cooperation of prehistoric and geomorphologic studies is of particular importance.

*) Anschrift des Verfassers: Dr. A. DittmanN, Geographische Institute der Universität Bonn, Meckenheimer Allee 166, D -5300 Bonn 1.
To exemplify the geomorpho-dynamic processes of this area during the last 30.000 years, it should be given a brief account of results obtained in the Wadi Deir at the southeastern slope of the Gebel-Galala-el-Qibliya. Within the area of investigation traces of former human occupation have been documented for middle paleolithic, neolithic and proto-historic times. They mostly include surface-finds of tools and stroke-places as well as rock-engravings. Moreover, sinter datings hint at the existence of a climatically favourable period at about 26.000 B.P., and ${ }^{14} \mathrm{C}$-datings of different charcoal layers prove human occupation for an abri, hollowed out within the younger wadi-sediments, for the time from 3.600 to 1.900 B. C.

\section{Einleitung}

Die Möglichkeiten und Grenzen eines Zusammenwirkens geomorphologischer und prähistorischer Arbeitsmethoden sind in den letzten Jahren verstärkt in den Vordergrund paläogeographischer Forschungsinteressen gerückt. Auf die Vorteile einer Zusammenarbeit zwischen Prähistorie und Physischer Geogaphie haben mehrfach ANDRES \& WUNDERLICH (1986), BEHRMANN (1950), BUTZER (1975), COPPENS (1969), DIMPLEBY (1975) sowie ROGNON (1976) hingewiesen. Die Vielseitigkeit interdisziplinärer Forschungsfelder - insbesondere in ariden und semiariden Gebieten - hat GABRIEL (1979) umfassend dargestellt.

Die Vorteile, aber auch die methodischen Beschränkungen einer paläogeographischen Interpretation prähistorischer Befunde sollen im folgenden kurz am Beispiel eines Untersuchungsgebietes im Norden der ägyptischen Eastern Desert vorgestellt werden. Die Forschungsergebnisse wurden im Rahmen eines von der DFG geförderten und von Prof. ANDRES, Marburg, geleiteten Projektes zur Untersuchung der jung- 
quartären Klima- und Reliefentwicklung dieses Raumes erarbeitet. Als die geomorphologischen Befunde bereits mehr oder weniger feststanden, kam es vor allem darauf an, Datierungshilfen - möglichst auch prähistorischer Art $-\mathrm{zu}$ finden (ANDRES 1987):

Die Gebirgsgegenden des nördlichen Teils der ägyptischen Eastern Desert sind gekennzeichnet durch eine Vielzahl flächenhaft verbreiteter, teilweise inein- andergreifender Systeme von Fußflächen und Wadisedimenten. Insbesondere die paläogeographische Rekonstruktion eines mehrfachen Wechsels von Erosions- und Akkumulastionsphasen kann hier wertvolle Hinweise auf frühere Klima- und Umweltbedingungen liefern.

Beispielhaft für das geomorphologische Geschehen dieses Raumes während der letzten 30.000 Jahre

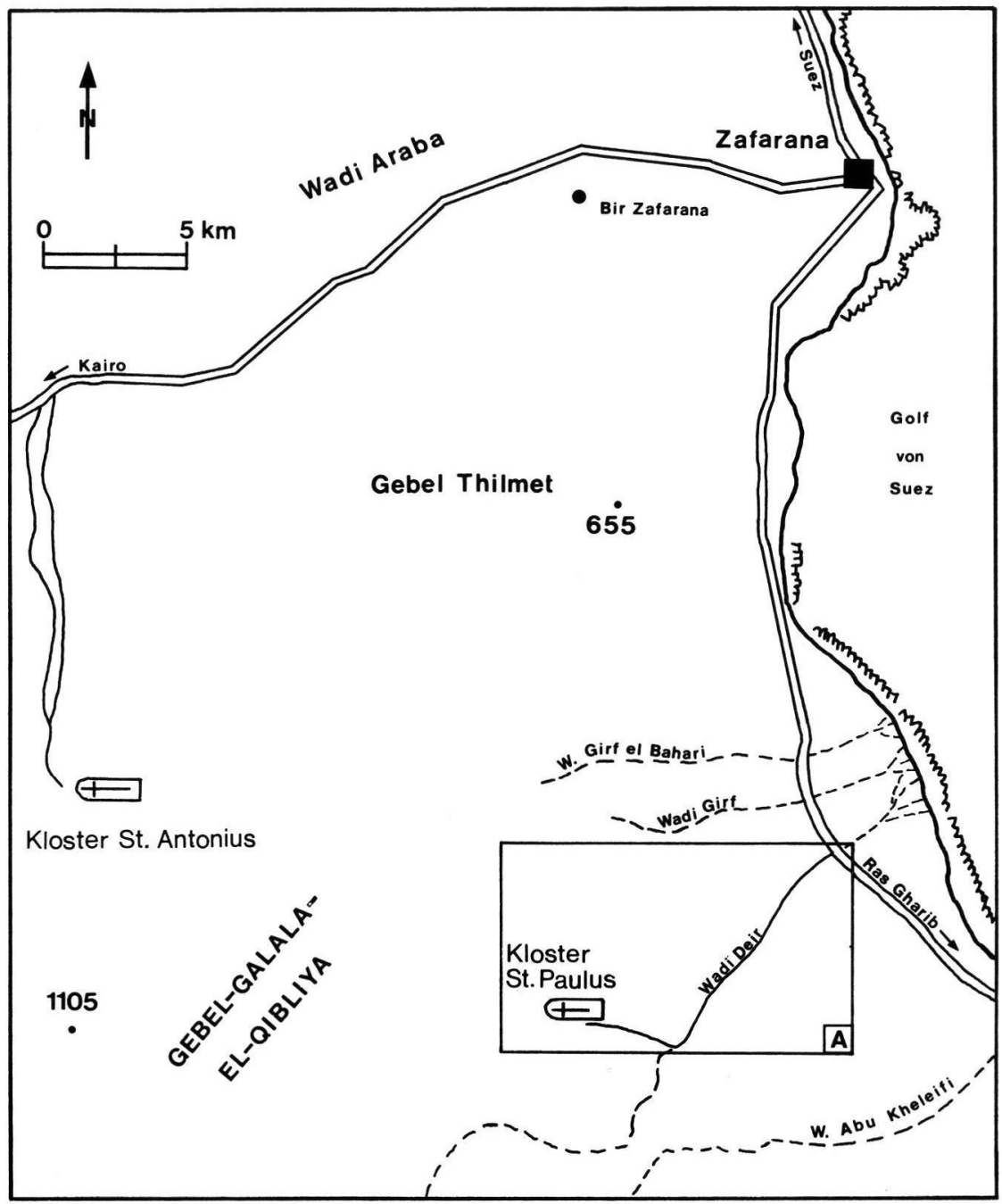

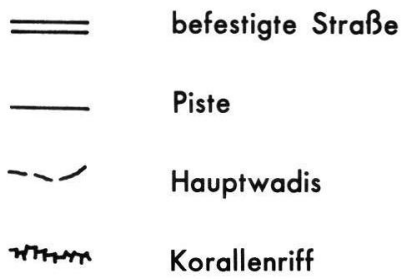

A
Abb. 1: Lage des Wadi Deir an der Südost-Abdachung des Gebel-Galalael-Qibliya.

Fig. 1: Location of Wadi Deir at the southeastern slope of Gebel-Galalael-Qibliya. 
wurden die Ergebnisse aus einem Untersuchungsgebiet an der SE-Abdachung des Gebel-Galala-elQibliya herausgegriffen. Es handelt sich dabei im wesentlichen um das Gebiet des Wadi Deir Bolos und dessen Einzugsbereich. Dieses Wadi ist auch auf unbeschrifteten Karten oder Satellitenbildern relativ leicht auszumachen, da es nicht, wie die meisten seiner Nachbarwadis in W-E-Richtung direkt dem Golf von Suez zustrebt, sondern sich - möglitherweise einer Verwerfungslinie folgend - von SW nach SE erstreckt. In die Untersuchungen einbezogen wurde auch ein von NW ins mittlere Wadi Deir einmündender größerer Seitenarm, der zur einfacheren Orientierung die Bezeichnung „Nordwadi” erhielt.

Im Bereich der Ostabdachung des Gebel-Galala-elQibliya sind in eozänen und kreidezeitlichen Kalken der Dachla-Serie zwei Steilstufen ausgebildet, die das Untersuchungsgebiet nach NW abgrenzen. Daran schließt sich nach $\mathrm{E}$ und $\mathrm{SE}$ zunächst der Bereich des Nubischen Sandsteins an. Er tritt im Nordwadi sowie im mittleren und obern Wadi Deir zu Tage. Miozäne und pliozäne Sedimente bilden den Übergang zum überwiegend mit quartären Schottern bedeckten küstennahen Gebiet und werden im mittleren und unteren Wadi Deir sichtbar.
Das Einzugsgebiet des Wadi Deir wird geprägt durch ausgedehnte Fußflächen älterer Schotter, die besonders in den mittleren und oberen Abschnitten der Wadis stark zertalt sind (Abb. 2). Insgesamt sind drei unterschiedliche Fußflächenniveaus erkennbar. Die beiden oberen Niveaus, die G1- und G2-Schotter, sind nicht mehr in allen Bereichen durchgehend vertreten. Dagegen bilden die darunter liegenden G3Schotter das Ausgangsglacis der jüngeren Zertalung. Die rezenten Abflußrinnen der Wadis sind in das Ausgangsglacis der Zertalung (G3) stark eingeschnitten. Sie folgen teilweise dem Verlauf älterer, im anstehenden Nubischen Sandstein angelegter Entwässerungssysteme. Diese Einschneidungen in Fußflächen und Nubischem Sandstein sind insbesondere in ihren oberen Bereichen durch die Sedimente einer jüngeren Talverfüllung gekennzeichnet, die im folgenden nach ANDRES (1987) als Hauptwadisediment (HWS) bezeichnet werden soll.

Auch diese Ablagerungen sind bereits weitgehend zertalt. Im Nordwadi sind sie noch weit verbreitet, im oberen Wadi Deir dagegen nur noch reliktisch erhalten. Aufbau und Struktur dieser Sedimente deuten darauf hin, daß das HWS durch reguläre fluviale Prozesse aufgebaut wurde (ANDRES 1987). Diese waren

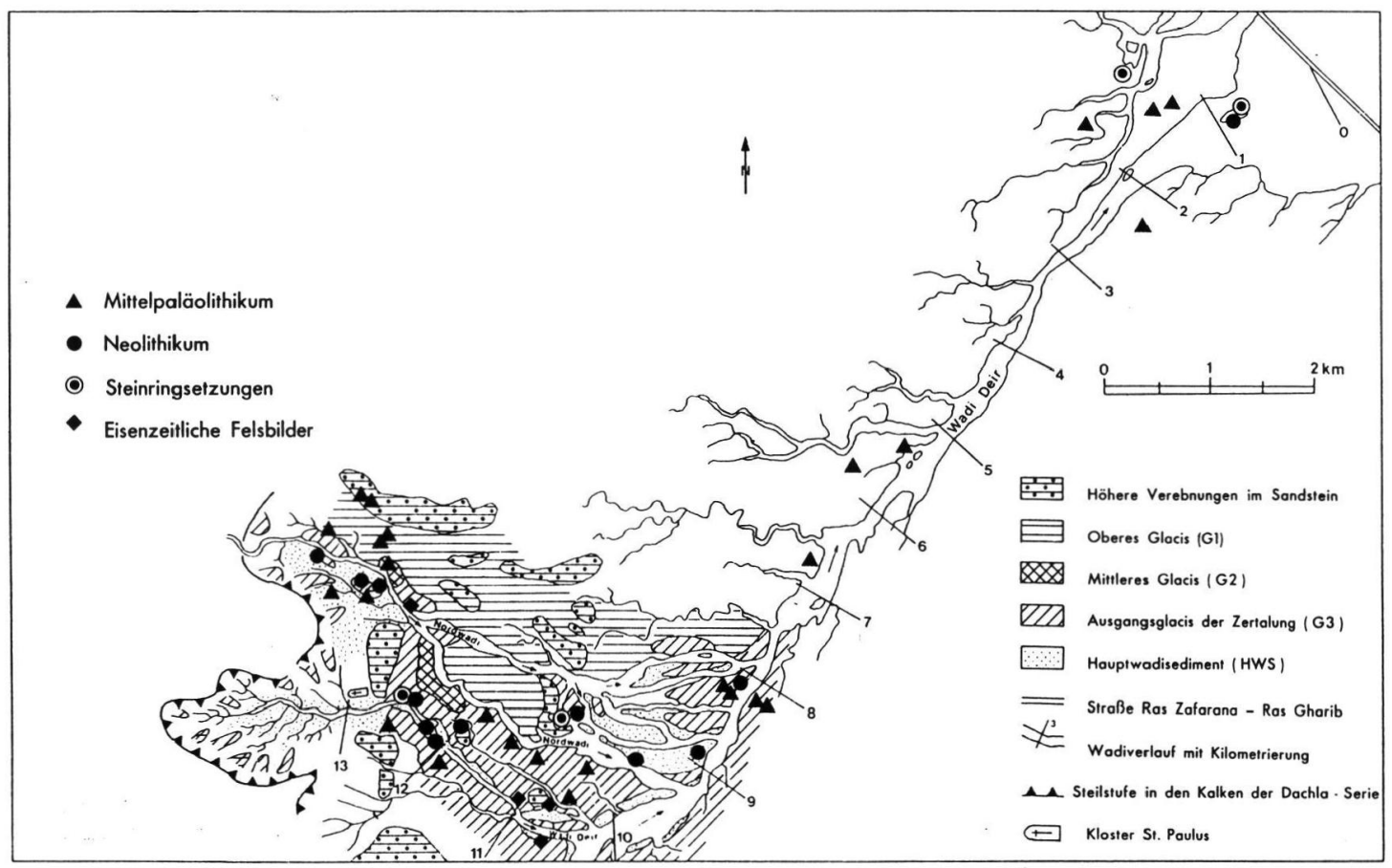

Abb. 2: Prähistorische Besiedlungsspuren und ihre Verteilung auf Fußflächen und Wadisedimenten im Gebiet des Wadi Deir.

Fig. 2: Traces of prehistoric occupation with regard to the distribution of pediments and wadi-sediments in the Wadi Deir region. 
gekennzeichnet durch eine extreme Überlastung sowie mit einem heftigen Abfluß beginnende und dann rasch abflauende Abkommen.

Das Abflußgeschehen unter den gegenwärtigen Klimabedingungen ist im Untersuchungsgebiet geprägt durch seltene episodische Einzelereignisse heftiger Abflüsse, deren Materialverlagerungen sich meist auf das aktuelle Wadibett beschränken. Eine nennenswerte Vegetation existiert kaum. Im Oberlauf des Wadi Deir wird eine kleine, gering schüttende Quelle von den Bauten des St. Paulus-Klosters umschlossen. Die besondere Lage, welche die Existenz dieser Quelle für das Wadi bedeutete, darf bei paläogeographischen Rekonstruktionsversuchen früherer Klima- und Umweltbedingungen anhand prähistorischer Besiedlungsspuren nicht außer Acht gelassen werden.

Spuren prähistorischer Besiedlung wurden im Gebiet des Wadi Deir vor allem aus dem Mittelpaläolithikum, dem Neolithikum und der zweiten Hälfte des ersten vorchristlichen Jahrtausends nachgewiesen. Dabei handelt es sich in erster Linie um Oberflächenfunde von Fertigwerkzeugen sowie Werk- und Schlagplätze.

\section{Die mittelpaläolithischen Funde}

Die mittelpaläolithischen Funde konzentrieren sich vor allem im Bereich der jüngeren Schotter des Ausgangsglacis der Zertalung (G3), kommen aber auch auf den älteren Niveaus der mittleren und oberen Fußflächen (G2 und G1) vor. Selten sind Funde im Bereich des anstehenden Nubischen Sandsteins. Auf dem HWS fehlt das Mittelpaläolithikum ganz. Für eine Rekonstruktion paläogeographischer Umweltbedingungen können die mittelpaläolithischen Funde nur bedingt herangezogen werden. Ihre Existenz belegt zunächst, daß zumindest der nördliche Teil der Eastern Desert während des Mittelpaläolithikums für Wildbeutergruppen durchgängig gewesen sein muß.

Die zahlreichen mittelpaläolithischen Fundstellen mit ungestörten Werk- und Schlagplätzen legen den Schluß nahe, daß es sich dabei nicht um verfrachtetes Material handeln kann. Insbesondere die Oberfläche der G3-Schotter scheint sich seit dem Mittelpaläolithikum kaum verändert zu haben. Abtragung ist hier allenfalls in Form einer Seitenerosion an den Randbereichen der tief eingeschnittenen Entwässerungssysteme vorstellbar. Auch dieser Befund erlaubt jedoch nur die Angabe eines Mindestalters für die drei bekannten Flußflächenniveaus. Erschwert wird dies durch das nur vage bestimmbare Ende des Mittelpaläolithikums in Nordostafrika (TAUTE 1978), so daß wir uns zunächst mit der groben zeitlichen Einstufung „,wahrscheinlich älter als 30.000 v. Chr." zufriedengeben müssen. Eine altersspezifische Differenzierung der Flußflächenniveaus G1, G2 und G3 untereinander ist anhand des prähistorischen Fundmaterials nicht möglich. Auch die zahlreich vorhandenen Levallois-Geräte (Abb. 3, Fig. 1 u. 2) können dieses Ergebnis nicht wesentlich präzisieren.

Erwähnenswert ist jedoch, daß sich die mittelpaläolithischen Funde im Bereich der G3-Schotter dort konzentrieren, wo dieses Niveau zur tiefer liegenden Oberfläche des Hauptwadisediments oder direkt zum aktuellen Wadiboden abfällt. Dies gilt besonders für die ausgedehnten Schlagplätze im mittleren Wadi Deir und unteren Nordwadi (Abb. 2). Eine solche Konzentration an den unmittelbaren Rändern der Taleinschnitte könnte ein Hinweis darauf sein, daß die Grundstruktur des heutigen Entwässerungssystems bereits zur Zeit des Mittelpaläolithikums angelegt war.

\section{Das Hauptwadisediment (HWS)}

Der geomorphologische Aussagewert der mittelpaläolithischen Funde drückt sich vor allem in der Bestimmung der Beziehungen zwischen den Niveaus des Hauptwadisediments (HWS) und des Ausgangsglacis der Zertalung (G3) aus: Die Tatsache, daß mittelpaläolithische Funde auf der Oberfläche des HWS nicht vorkommen, ist ein Hinweis darauf, daß diese Ablagerungen zur Zeit des Mittelpaläolithikums noch nicht ausgebildet waren. Sehr wahrscheinlich entstand das HWS erst in post-paläolithischer Zeit. Insbesondere die sedimentologischen Ergebnisse (ANDRES 1987) sprechen dafür, daß diese Ablagerung nach dem Ende einer länger andauernden feuchten Klimaphase erfolgte: Die das HWS aufbauenden Lockerund Feinmaterialien bestehen zu $95 \%$ aus Kalken des oberen Einzugsbereichs der Wadis, während der im Ablagerungsgebiet anstehende Nubische Sandstein kaum vertreten ist. Dieses Material fand während einer Feuchtphase auch im Bereich der Hanglagen Halt unter einer schützenden Vegetationsbedeckung. Die später einsetzende Austrocknung des Raumes besaß den Charakter einer ökologischen Katastrophe. Die schützende Pflanzendecke verschwand, die Lockermaterialien waren der Erosion preisgegeben und gerieten in Bewegung. Ihre Ablagerung erfolgte in den oberen und mittleren Bereichen der tief in die G3-Schotter eingeschnittenen Entwässerungssysteme. Die teilweise bis zu $15 \mathrm{~m}$ mächtigen Talsedimente erreichten jedoch nirgendwo das Niveau der älteren G3-Schotter.

Hinweise auf eine vor der Ablagerung des HWS existierende Feuchtphase liefert neben den Relikten 


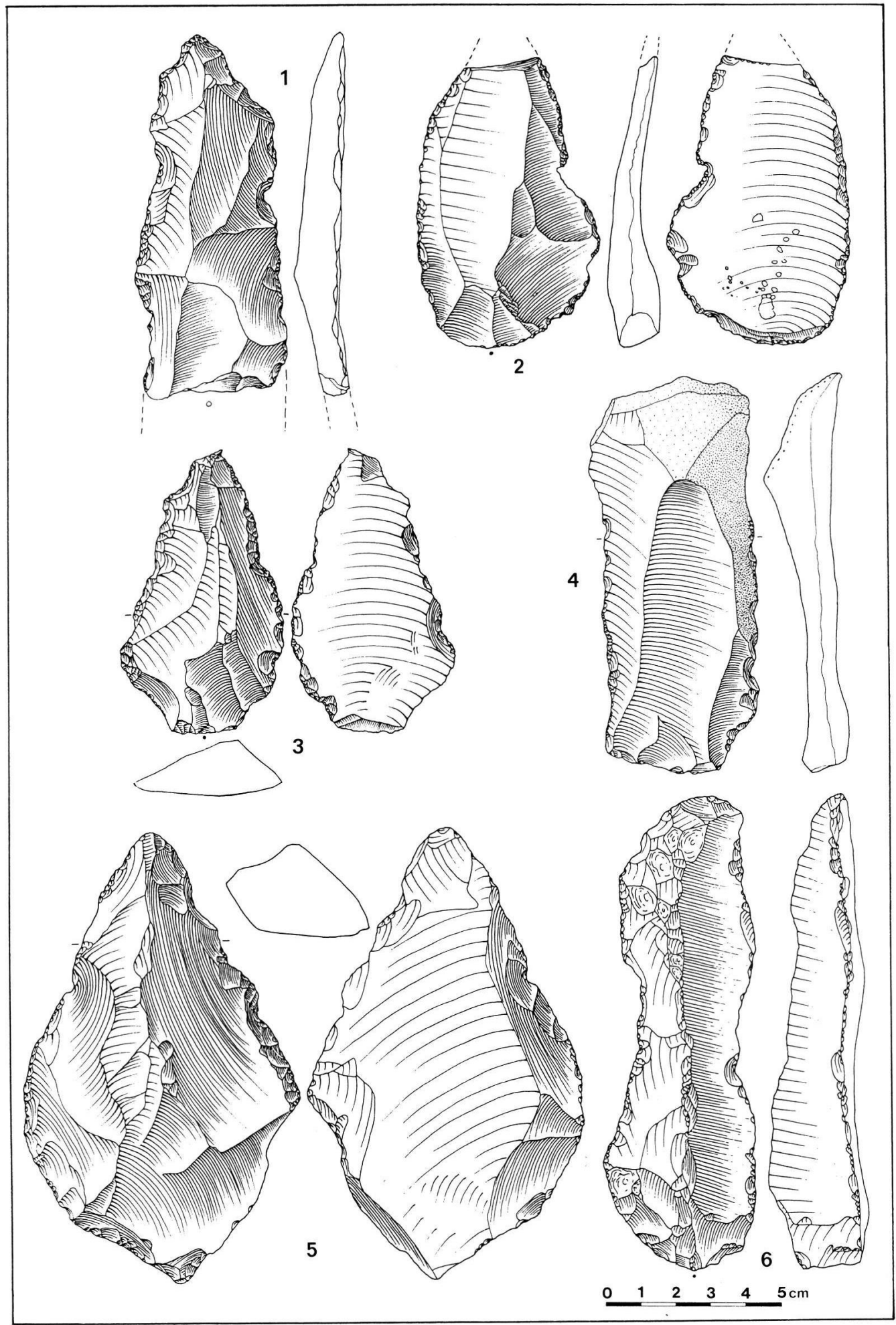

Abb. 3: Mittelpaläolithische Artefakte aus dem Wadi Deir.

Fig. 3: Middle paleolithic artifacts from Wadi Deir. 
einer früheren Bodenbildung auf den G3-Schottern auch die Bildung von Kalksinter. Die Sinter entstanden zu einer Zeit, als die Quelle im Bereich des heutigen Klosters so stark schüttete, daß offene Wasserflächen und Sinterterrassen entstanden. Sinterproben wurden im oberen Wadi Deir unterhalb des St. Paulus-Klosters entnommen. Die ermittelten ${ }^{14} \mathrm{C}$-Werte liegen bei 26.350 und 26.900 B.P. (HD 7495-8189 und -8192). Außerdem wurden im Nordwadi stark übersinterte ältere Schotter gefunden und mit 26.000 B.P. (HD 7946-8220) datiert. *) Die Fundlage zeigt, daß die Übersinterung stattfand, als das Ausgangsglacis der Zertalung (G3) bereits stark zerschnitten war. Zu diesem Zeitpunkt traten an der Basis der G3-Schotter reichlich kalkhaltige Wasser aus. Biogenes $\mathrm{CO}_{2}$ war in überwiegendem Maße an der Kalkausscheidung beteiligt, so daß von der Existenz einer relativ dichten Vegegationsbedeckung im oberen Wadibereich ausgegangen werden kann.

Die Sinterdatierungen liefern lediglich Hinweise auf die Existenz einer klimatischen Gunstperiode um 26.000 B. P.. Wann diese Feuchtphase begann bzw. wie lange sie vor dem faßbaren Zeitraum von 26.000 B.P. bereits andauerte und wann sie endete, geht aus diesen Befunden nicht hervor. Es kann daher auch nicht mit Sicherheit entschieden werden, ob die mittelpaläolithischen Funde zur gleichen oder zu einer anderen, früheren Feuchtphase gehören. Es muß betont werden, daß die unmittelbar nach der Ablagerung des HWS einsetzende Wiedereinschneidung erfolgte, ohne daß eine weitere Klimaänderung stattgefunden hätte. Auch die Zerschneidung des HWS fand unter ariden Klimabedingungen statt. Nachdem die Hänge größtenteils bis zum Anstehenden freigelegt waren, führte die ausbleibende Zufuhr weiteren Erosionsmaterials zur übergangslos einsetzenden Zerschneidung des HWS im Zuge episodischer oder periodischer Wadiabkommen.

\section{Neolithische Datierungshilfen}

Über den Zeitraum nach der Ablagerung und Wiederzerschneidung des HWS geben die neolithischen Funde Aufschluß. Ihr Vorkommen auf der Oberfläche dieser Ablagerungen beweist, daß das HWS zur Zeit des Neolithikums bereits bestanden haben muß. Darüberhinaus belegen Funde in Einschnitten und Ausbuchtungen dieses Sedimentkörpers, daß auch seine Wiedereinschneidung im Neolithikum bereits weitgehend abgeschlossen war. Die neolithischen Funde

*) Die Datierungen wurden dankenswerterweise von Herrn Dr. W. KROMER vom Institut für Umweltphysik der Universität Heidelberg durchgeführt. konzentrieren sich vor allem auf die Oberfläche des HWS, kommen jedoch auch auf den G3-Schottern noch häufiger vor. Im Bereich der höheren Niveaus und des anstehenden Nubischen Sandsteins treten sie insbesondere dort auf, wo günstige Geländepositionen einen weiteren Ausblick ermöglichten.

Von besonderer Bedeutung für eine relative Datierung von Ablagerung und Wiederzerschneidung des HWS ist eine spätneolithische Fundstelle im oberen Wadi Deir auf der rechten Talseite. Es handelt sich dabei um die Relikte eines verstürzten Abris, dessen heruntergebrochene Decke Teile von Siedlungsschichten konservierte. Dieses Abri war in den im oberen Wadi Deir bis zu 13 m mächtigen HWS-Ablagerungen ausgebildet. Die Fundstelle ist gekennzeichnet durch eine markante, säulenartig aufragende Sedimentformation. Diese weist die für das HWS charakteristischen horizontalen Schichten auf. Die Verbindung zwischen der Sedimentsäule und den Ablagerungen am Talrand bilden zwei Halden aus gelocker-

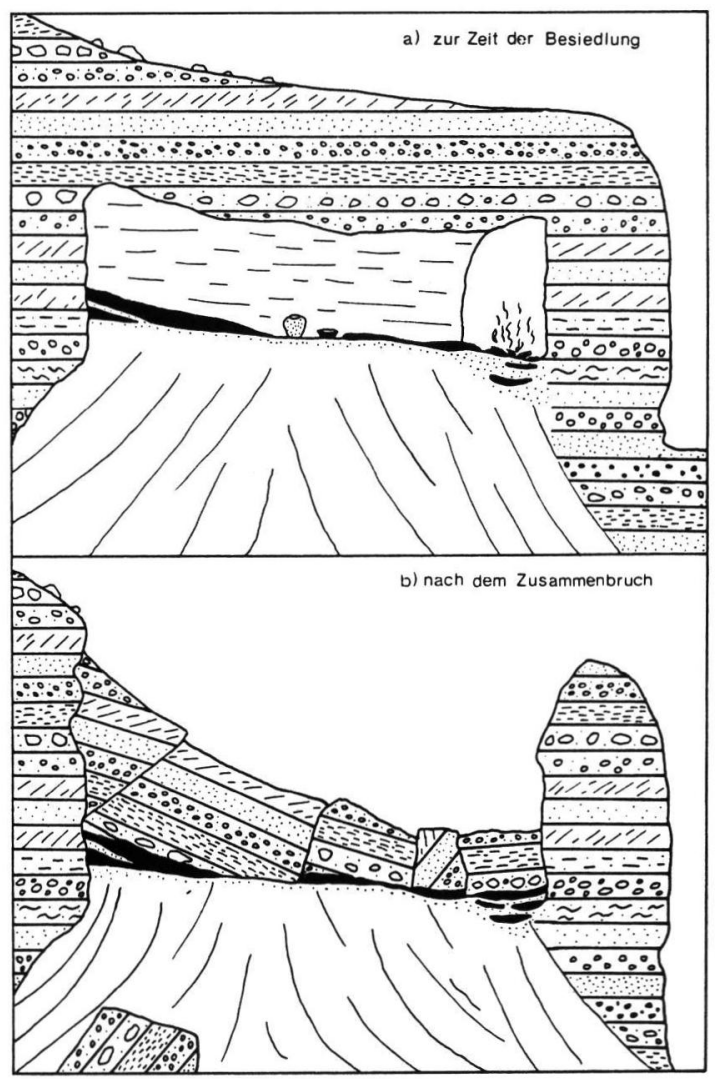

Abb. 4: Rekonstruktionsversuch des neolithischen Abris im oberen Wadi Deir.

Fig. 4: Reconstruction of the neolithic abri in upper Wadi Deir. 
tem und verfrachtetem HWS-Material. Es handelt sich dabei um die Überreste der heruntergebrochenen ehemaligen Abri-Abdachung. Von den beiden, dachförmig einander zugeneigten Halden ist die eine nach NO, die andere nach SW ausgerichtet.

Aufgefallen war die Fundstelle durch frei erodierte und verfrachtete Holzkohleteile und Tierknochenfragmente. Leider stellte sich sehr bald heraus, daß das organische Material nicht - wie zunächst erhofft - bei der Ablagerung des HWS mit eingebaut worden war. Die einzelnen Fundschichten wurden erst sehr viel später in Einschnitten und Ausbuchtungen des bereits bestehenden HWS angelegt. Damit war klar, daß eine Datierung der Fundstelle keinen Aufschluß über den Zeitraum der Ablagerung des HWS erbringen konnte, sondern lediglich die Angabe eines Mindestalters für dessen Wiederzerschneidung.

Schon zur Zeit der Besiedlung des Abris muß die Fundstelle etwa dem heutigen Aussehen entsprochen haben: Die Fundschichten der Westseite werden von größeren, verfestigten Sedimentblöcken überlagert. Diese zeigen allerdings nicht die für das HWS kennzeichnende Horizontallagerung, sondern sie sind aus ihrer ursprünglichen Position abgerutscht. Es kann daher als wahrscheinlich gelten, daß hier eine im HWS ausgebildete Überdachung als Lagerplatz dien- te. In unmittelbarer Nähe der Sedimentsäule deutet eine Vielzahl stratigraphischer Hinweise darauf hin, daß einst eine durchgehende Verbindung zwischen der Ost- und der Westseite bestand. Entweder war die markante Sedimentsäule bereits damals schon vom übrigen Sedimentkörper isoliert oder es bestand eine torbogenartige Verbindung zu den talrandwärts liegenden Ablagerungen (Abb. 4).

Für fünf Siedlungsschichten der Fundstelle liegen ${ }^{14} \mathrm{C}$-Datierungen vor. Nach einer dendrochronologischen Umsetzung dieser Daten bilden sich deutlich folgende vier unterschiedlichen Besiedlungsphasen heraus (Abb. 5): *)

In die erste Phase zwischen etwa 3.630 und 3.365 B.C. gehört die insgesamt fundarme Schicht WR-4 auf der Westseite. Die zweite Phase zwischen 3.050 und 2.695 B.C. ist insbesondere durch zahlreiche Tierknochenfragmente (Nubischer Wildesel, Steinbock, Schaf, Ziege) gekennzeichnet. Kurz danach folgt zwischen 2.660 und 2.390 B. C. die dritte Besiedlungsphase mit Fundschichten auf der Ostseite,

*) Die Datierungen wurden dankenswerterweise von Herrn Prof. Dr. M. A. GeYH vom Niedersächs. Landesamt für Bodenforschung in Hannover durchgeführt.

\begin{tabular}{|c|c|c|c|c|}
\hline $\begin{array}{l}\text { Jahre } \\
\text { v.Chr. }\end{array}$ & \multicolumn{2}{|c|}{$\begin{array}{l}\text { Besiedlungs- } \\
\text { phasen }\end{array}$} & Westseite & Ostseite \\
\hline 2.000 & $\begin{array}{l}1.920 \\
2.150\end{array}$ & IV. & -- & $\frac{W R-1}{W R-2}-\cdots-1$ \\
\hline \multirow[t]{2}{*}{2.500} & $\begin{array}{l}2.390 \\
2.660\end{array}$ & III. & \multicolumn{2}{|c|}{$\begin{aligned} \mathrm{WR}-2,5 \longrightarrow \mathrm{WR}-3 \longrightarrow \mathrm{OL}-1 \\
\mathrm{OL}-2\end{aligned}$} \\
\hline & $\begin{array}{l}2.695 \\
3.050\end{array}$ & II. & $\begin{array}{l}W L-1 \leftrightarrow \text { WM-1 } \\
W L-2 \quad \text { WM-2 }\end{array}$ & OL-3 \\
\hline 3.500 & $\begin{array}{r}3.365 \\
3.630 \\
\end{array}$ & I. & & WR-4 \\
\hline 4.000 & & & & \\
\hline
\end{tabular}

Abb. 5: Die nachweisbaren Besiedlungsphasen des Abris und ihre Verteilung auf Ost- und Westseite.

Fig. 5: Documented periods of occupation and their position at the western and eastern part of the abri. 
die stratigraphische Verbindungen mit der Westseite des Abris aufweisen. Die letzte nachweisbare Besiedlung des Abris fällt in den vierten Zeitabschnitt zwischen ca. 2.150 und 1.920 B. C..

Über einen Zeitraum von mehr als 1.600 Jahren hinweg wurde das Abri episodisch immer wieder aufge- sucht und als Lagerplatz benutzt. Dies zeigt, daß die äußere Struktur des Hauptwadisediments während dieser Zeit zumindest im Bereich des oberen Wadi Deir weitgehend unverändert geblieben sein muß. Der endgültige Zusammenbruch des Abris erfolgte sehr wahrscheinlich unmittelbar nach dem Ende der vierten und letzten Besiedlungsphase.
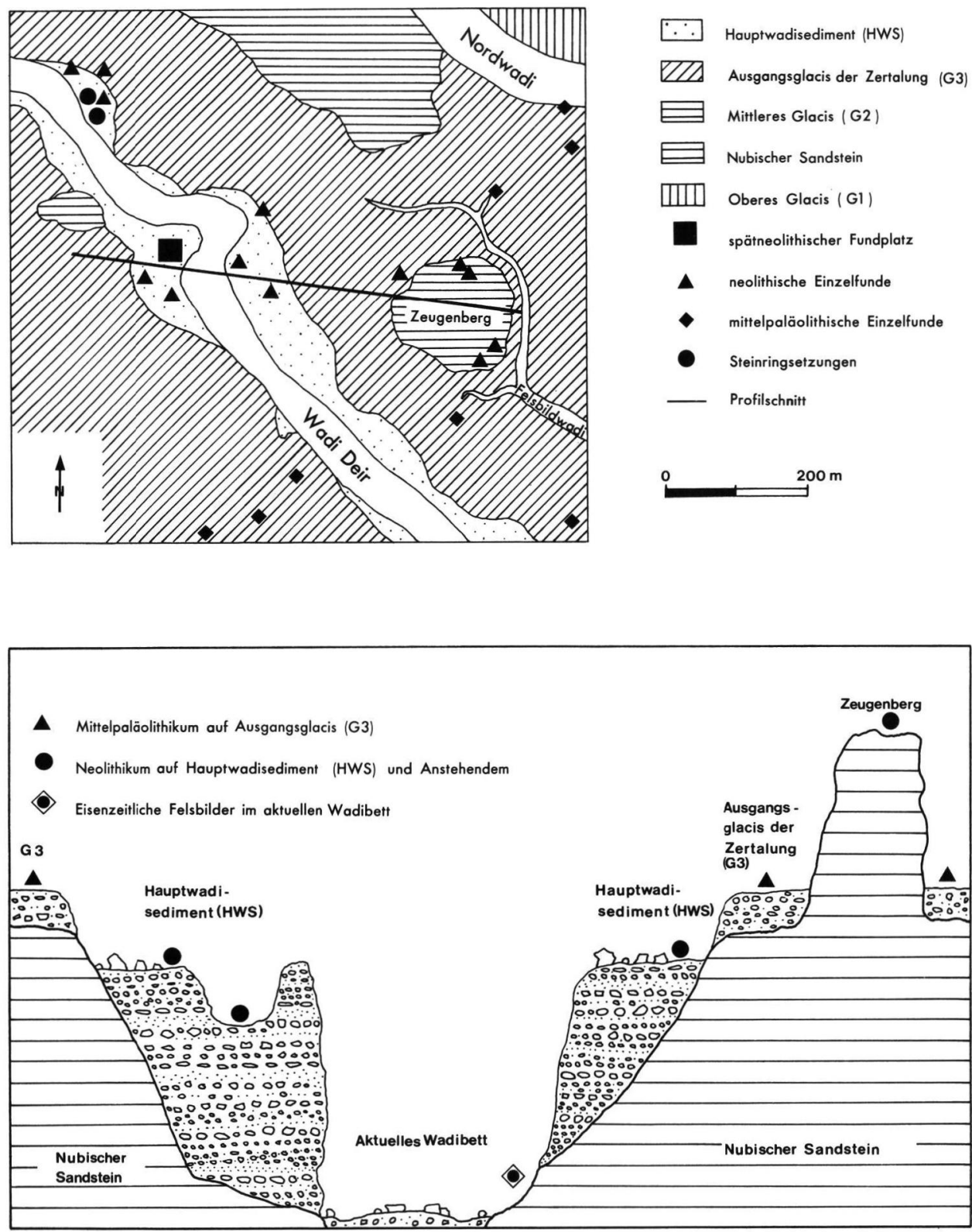

Abb. 6: Die prähistorischen Besiedlungsspuren des oberen Wadi Deir (oben: Lage des Profilschnittes, unten: stark überhöhte Profilzeichnung).

Fig. 6: Traces of prehistoric occupation from upper Wadi Deir (above: location of cross section, down: super-elevated cross section). 
Die Lage der ältesten Fundschicht verdeutlicht, daß das HWS um etwa 3.700 B.C. mindestens bis zur Höhe dieser Schicht zerschnitten gewesen sein muß. Sie befindet sich heute 5,3-6,5 m über dem Niveau der rezenten Abflußrinne. Auf der weiter talaufwärts gelegenen Ostseite des Abris deutet die Position der Fundlagen darauf hin, daß die Ablagerungen möglicherweise noch weitaus tiefer zerschnitten gewesen sein könnten. Unter Berücksichtigung der Annahme, daß die spätneolithischen Lagerplätze nicht in gleicher Höhe mit der damaligen Abflußrinne angelegt wurden, kann gefolgert werden, daß das HWS während der ersten Besiedlungsphasen des Abris mindestens bis zu einer Höhe zerschnitten war, die etwa 1,5 bis 3,0 $\mathrm{m}$ über dem rezenten Niveau gelegen haben dürfte.

\section{Schlußbemerkungen}

Die stark überhöhte Profilzeichnung eines Querschnitts im oberen Wadi Deir veranschaulicht eine Zusammenfassung der bisherigen Ergebnisse (Abb. 6): Deutlich wird die Verteilung der mittelpaläolithischen Fundlagen und die Konzentration der neolithischen Besiedlungsspuren auf den Bereich des Hauptwadisediments sowie herausragende Landmarken. Ebenfalls eingetragen ist die Lage postneolithischer Felsgravierungen, deren Ausrichtung zeigt, daß seit der 2. Hälfte des 1. vorchristlichen Jahrtausends im Bereich des Wadi Deir keine wesentlichen Niveauveränderungen stattgefunden haben.

Die Darstellung der Untersuchungsergebnisse aus dem Gebiet des Wadi Deir zeigt, daß eine Vielzahl paläogeographisch-paläoklimatischer Fragen erst durch ein interdisziplinäres Zusammenwirken geomorphologischer und prähistorischer Arbeitsansätze einer befriedigenden Beantwortung zugeführt werden kann. Eine isolierte Betrachtung der jeweiligen Einzelergebnisse wäre allenfalls im Stande, ein überaus fragmentarisches Bild zu vermitteln. Andererseits liegen die Beschränkungen einer Zusammenarbeit dort, wo für eine relative zeitliche Einordnung insbesondere älterer Oberflächenformen die Differenzierungen des prähistorischen Materials nicht ausreichen, um Abstufungen herausarbeiten $\mathrm{zu}$ können. Es lassen sich jedoch keineswegs alle vor- und frühgeschichtlichen Ergebnisse auf geomorphologische Sachverhalte übertragen. Im Überschneidungsbereich beider Wissenschaften ist es die Aufgabe der Paläogeographie, einen groben zeitlichen Rahmen früherer Klima- und Umweltbedingungen abzustecken, den mit Inhalt zu füllen der Prähistorie obliegt.

\section{Schriftenverzeichnis}

ANDRES, W. (1987): Geomorphodynamik und Reliefentwicklung in der nördlichen Eastern Desert in den letzten 30.000 Jahren. - Verhandlungsbd. Dt. Geographentag in Berlin: 183-188; Berlin.

— \& WUNDERLICH, J. (1986): Untersuchungen zur Paläogeographie des westlichen Nildeltas. - Marburger Geogr. Schr., 100: 117-131; Marburg.

Behrmann, W. (1950): Die Bedeutung der Geographie für die Prähistorie. - Geogr. Rundschau, 2: 168-174; Braunschweig.

BUTZER, K. W. (1975): The ecological approach to archeology: Are we really trying? - American Antiquity, $\mathbf{4 0}$ (1): 106-111; Washington D.C.

COPPENS, Y. (1969): De l'Archéologie à la Paléogéographie. - Bull. Inst. Franc. Afrique Noire, A, 31 (1): 263268; Dakar.

Dimbleby, G. W. (1975): Archeological evidence of environmental change. - Nature, 256: 265-267; London.

GABRIEL, B. (1979): Ur- und Frühgeschichte als Hilfswissenschaft der Geomorphologie im ariden Nordafrika. Stuttgarter Geogr. Studien, 93: 135-148; Stuttgart.

RoGNON, P. (1976): Essai d'interprétation des variations climatiques au Sahara depuis 40.000 ans. - Rev. Géogr. phys. et Géol. dnyamique, 18: 251-282; Paris.

TAuTe, W. (1978): Das Ende der Altsteinzeit in Nordafrika. - Museen der Stadt Köln (Hg.): Sahara 10.000 Jahre zwischen Weide und Wüste: 48-59; Köln.

Manuskript eingegangen am 13.9. 1989,

Nachträge März 1990. 